\title{
Micro RF sensor applied in grid radio monitoring system
}

\author{
XU Shi-min ${ }^{1, a}$, YU Hong-bing ${ }^{1, b}$ and CHU Fei-huang ${ }^{1, c}$ \\ ${ }^{1}$ Electronic Engineering Institute, Hefei, China \\ axushimin@126.com, bjipinwu@sina.cn, chufeihuang@163.com
}

\begin{abstract}
Keywords: Grid radio monitoring system, Micro radio frequency(RF) sensor, Digital receiver Abstract: Grid radio monitoring system is composed of dense radio sensors in a certain area, and the nodes are interconnected and shared, so the overall cognition performance of the electromagnetic environment is improved. Micro RF sensor is the core equipment in the system, so this paper constructs a micro RF sensor system, and analyzes the key technology of its implementation. Test result shows that the RF sensor has a good cost performance and a broad application prospect.
\end{abstract}

\section{Introduction}

With the development of radio technology, radio technology has been widely used in every field of the society and people's daily life. Radio technology application field has covered the radio and television departments of radio and TV Broadcasting Coverage System, public security and military departments of trunked communication and command and communication system, civil aviation navigation and communication system, maritime and fishery sector of communication station, railway and urban rail transit dispatch and command and emergency communication system, meteorological departments in all kinds of weather detection radar and weather warning transmitter, and so on. Spectrum resource contradiction between supply and demand has become increasingly prominent and the electromagnetic environment is becoming more and more complex. Radio monitoring and management department not only executes the daily monitoring and management of security tasks, but also needs to find a large number of illegal radio stations and various types of interference from base station, aviation, railway and other industries.

The contradiction between the growing demand of wireless communication technology and the existing radio monitoring technology is becoming more and more prominent. Radio monitoring, especially in large cities, is facing a great challenge. How to manage the radio spectrum resources more scientific, standardized and precise has become a difficult and important problem in the present and future radio management.

With the development of information technology such as Internet of things, big data, cloud computing and so on, it also makes the wireless communication more difficult to be monitored. Grid radio monitoring and management system changes traditional radio monitoring network mainly rely on fix and large stations. Under the support of the integrated information platform, it uses modern information processing technology to establish rule and business process across department management, to achieve a high degree of automatic business processing and monitoring resource sharing mechanism, achieves the intelligent analysis of monitoring data, intelligent distribution monitoring tasks, intelligent allocation of frequency resources, intelligent electromagnetic spectrum measured and comprehensive and dynamic control, to enhance the radio monitoring and comprehensive management ability. 


\section{Brief introduction of grid radio monitoring system}

Using low cost RF sensors densely arranged in certain regions, grid radio monitoring system can form a grid monitoring network, as close as possible to the coverage area within the transmitter, so as to remove the bad influence of higher carrier frequency and wider bandwidth of radio monitoring. Typical grid radio monitoring system framework is shown in the following diagram.

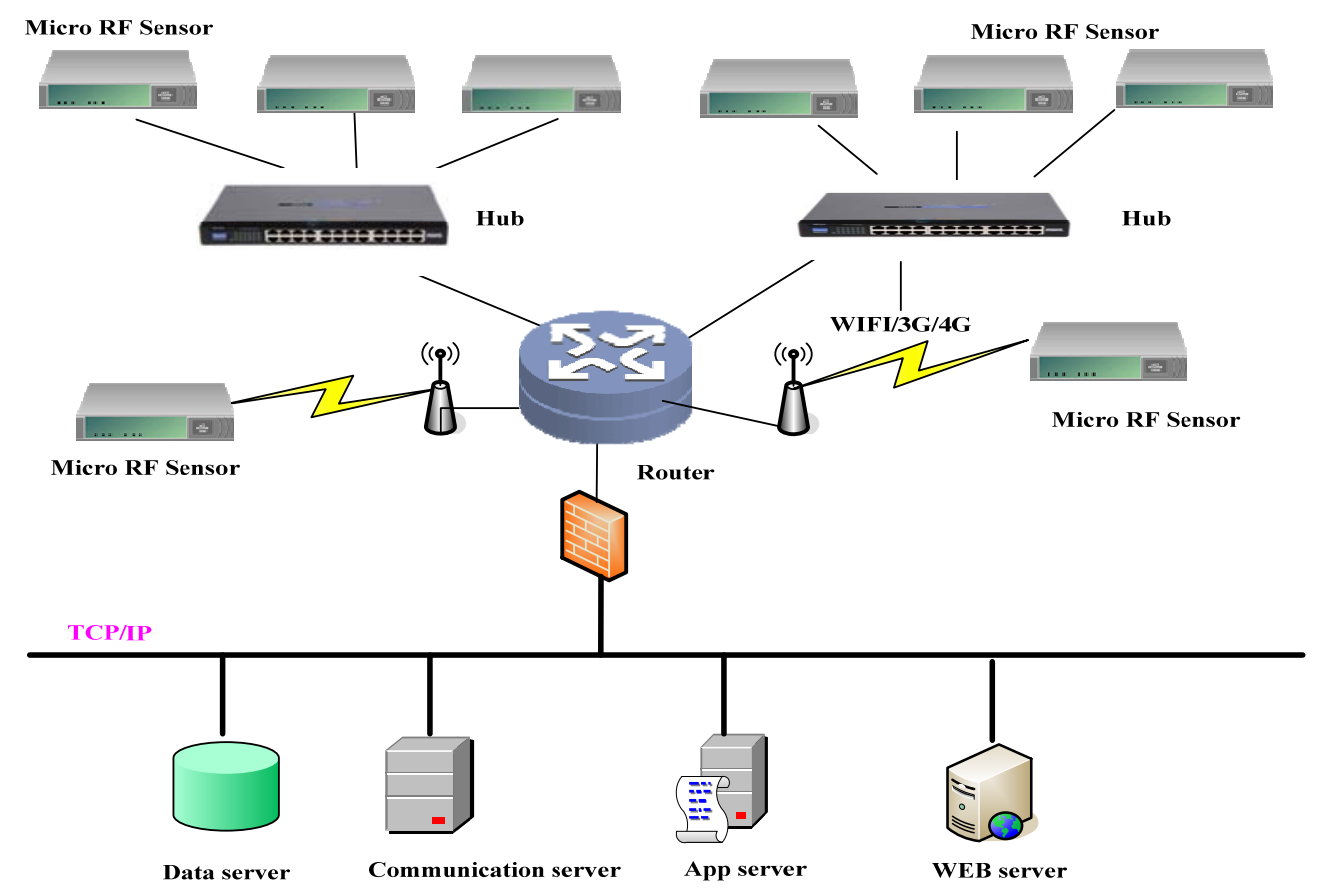

Fig.1 Grid radio monitoring system architecture diagram

Grid radio monitoring technology takes advantage of better data exchange and information sharing to improve the performance of radio spectrum monitoring. Large number of micro size and high performance receivers are needed in the grid radio monitoring system, but traditional receiver is difficult to solve the problems such as cost, power consumption, volume and weight at present. Therefore, this paper will research the development of micro RF sensors of low cost, low power consumption, large bandwidth and integration monitoring functions, to establish a grid monitoring management demonstration applications, and promote the development of radio monitoring.

\section{System scheme of micro RF sensor}

Micro RF sensor in the technical principle is equivalent to broadband digital radio receiver, further system integration in the portable product, uses large-scale monolithic system integration (SOPC) technology in high speed digital processing and control part, makes the traditional sense of the radio receiver evolved into micro broadband RF sensor, and its application field is greatly expanded.

The principle of micro RF sensor is adopted as the structure of the super heterodyne architecture shown in figure 2 The input RF signal is filtered, using a mixer and a local oscillator(LO) to lower the frequency to the intermediate frequency(IF). In order to overcome the limitation of scanning time and so on, the receiver architecture uses digital intermediate frequency, and performs IF filtering in the digital domain. Compared with analog filter, digital filter can greatly improve the scan time. Digital signal processing (DSP) in the IF is a very convenient method for the analysis and identification of the engineers. The sensor has two antenna inputs, which are used to connect to a local broadband or a diversity antenna. The system also includes a set of strip line pre-selection 
filters. These optional filters are designed to reduce the cost of the receiver and improve the reliability of the search for small signal in high power transmission is very useful. Digital frequency converter(DDC) is used to low the frequency to baseband, with up to $20 \mathrm{MHz}$ of variable bandwidth, can adapt to a variety of wireless technology and modulation types. Embedded software controls trigger, FFT operation and memory acquisition. The sampled data with time stamp is transmitted to a remote server through the network, and the signal identification and data record is performed by the server.

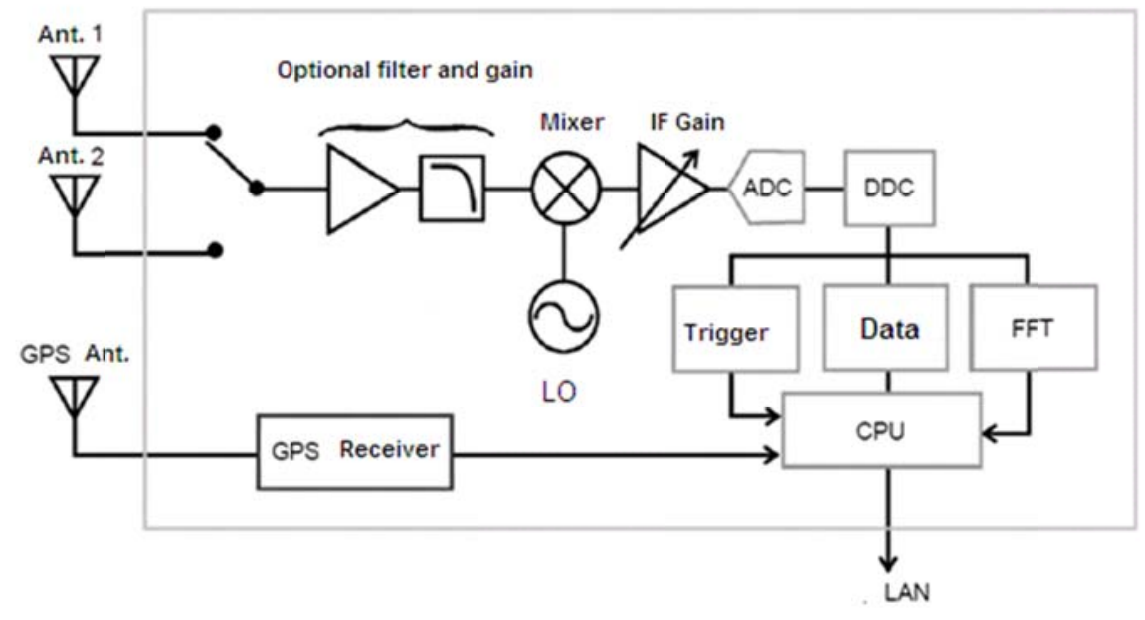

Fig.2 Micro RF sensor principle block diagram

\section{Key technology of micro RF sensor}

Based on the FPGA SOPC digital intermediate frequency processing technique is the first key technology. IF digital receiver is an important technical means, not only can improve the performance, stability and reliability of the product, but also can reduce the volume, reduce the cost of the instrument. After high speed A/D, the data is stored in the FPGA, and the data of a time span is stored in the memory of the large capacity. The signal processing and controlling system based on SOPC DSP and ARM are integrated into a single chip, so that the system level tasks can be accomplished in a single chip. The principle diagram is shown in figure 3

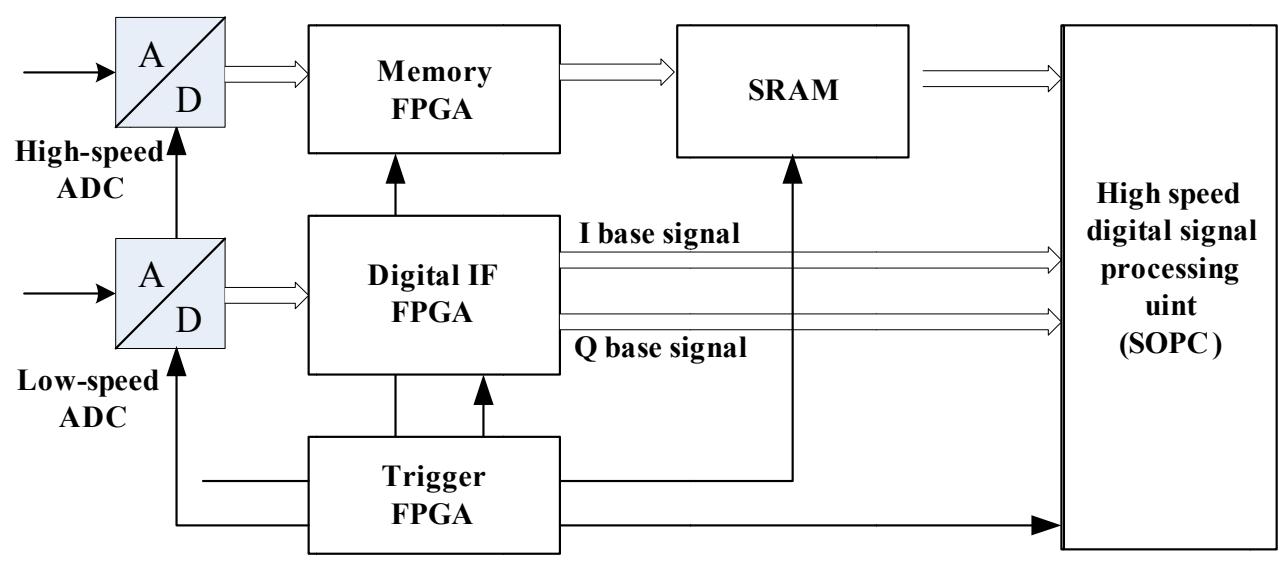

Fig.3 Digital IF signal processing principle block diagram

$70 \mathrm{MHz}$ IF output to the A/D 16bit converter for digital, A/D using the clock is $100 \mathrm{MHz}$. Digital IF data flow is divided into two parts by digital multiplier respectively, and a local oscillator digital produce cos components and the sin of component are multiplied, the input signal in the frequency domain of the shifting, carrier frequency is zero, then entered the CIC digital low pass filter, and the 
corresponding extraction, and then after FIR filter, two baseband signal of in-phase component and quadrature component are obtained. As the signal bandwidth is reduced, the Q and I signals can be further extracted by CIC and FIR filters. Its relationship is as follows:

$$
s(n)=x_{I}(n) \cos \omega_{c} n+x_{Q}(n) \sin \omega_{c} n
$$

Among them, $x_{I}(n)$ is the phase components and $x_{Q}(n)$ is the orthogonal components of the signal respectively. $\omega_{c}$ is the carrier frequency of the input IF signal. The frequency conversion is achieved by multiplying the signal $s(n)$ with the digital oscillator $\left(\cos \omega_{c} n-j \sin \omega_{c} n\right)$ :

$$
\begin{aligned}
& s(n)\left(\cos \omega_{c} n-j \sin \omega_{c} n\right) \\
& =\frac{1}{2}\left[x_{I}(n)+x_{I}(n) \cos 2 \omega_{c} n-j x_{Q}(n) \sin 2 \omega_{c} n+x_{Q}(n) \sin 2 \omega_{c} n-j x_{Q}(n)+j x_{Q}(n) \cos 2 \omega_{c} n\right]
\end{aligned}
$$

Afer the digital low pass filter can be filtered by the two harmonic components, the two baseband signals can be obtained:

$$
x(n)_{L P F}=\frac{1}{2}\left[x_{I}(n)-j x_{Q}(n)\right]
$$

Due to the requirements of the measurement speed, digital mixing, CIC filtering and FIR filtering are used in hardware.

After digital mixing, the signal data flow into the signal analysis, first of the resolution bandwidth filter with linear phase Gauss low-pass filter, resolution bandwidth from $10 \mathrm{~Hz}$ to $10 \mathrm{MHz}$ step can be adjusted. Subsequently, the signal can be entered into the FFT processing module and the general amplitude calculation module. At the sweep frequency measurement mode, the amplitude the I and Q signals of modulus is acquired, and after filtering and video digital demodulation spectral data is obtained in swept frequency range. In addition, in the FFT mode, the I and Q signals are compensated by amplitude compensation, and the compensation method is based on a digital filter with the amplitude frequency characteristics. After complex FFT transform, the complex spectrum signal is obtained, and then the spectrum data is obtained within the maximum bandwidth.

Tunable radio frequency band-pass filtering technology is the second key technology. For the receiver, the out of band and mirror response will seriously affect the measurement function and even make the equipment can not be used normally, so they must be effectively suppressed. The input signal to be measured before the first mixer is added to a set of programmable tunable RF bandpass filter. The control system switches automatically into the right filter branch based on the signal frequency band. The system can effectively suppress the band and mirror response, and can effectively improve the dynamic range of the three degree of the two harmonic distortion. In addition, in order to ensure that the signal does not produce a large distortion after the frequency conversion filter, it is required that the pass band ripple of the filter, group delay, and the insertion loss is small enough.

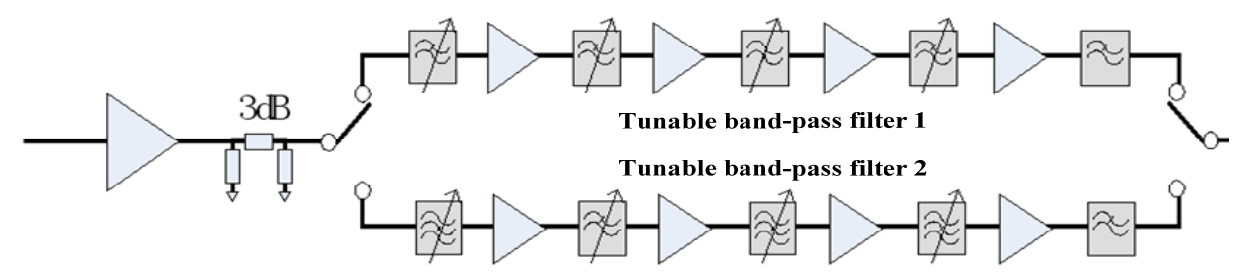

Fig.4 Tunable filtering principle block diagram 
Figure 4 shows the need to track the radio frequency input signal processing, and then mixed, with two switches can be tuned band pass filter. You can tune with adjustable band pass filter 1 or 2 to suppress the harmonic frequency of the input signal, the three order distortion and image frequency signal, each filter is a variable frequency, relative bandwidth of micro strip band pass filter, and can be controlled by the preset data.

The tracking and filtering function is realized by using the multistage adjustable harmonic oscillators. As shown in Figure 5, each oscillator is tuned at the corresponding suppression frequency. If two oscillators are tuned at the mirror frequency point, and another oscillator is tuned at the two harmonic frequency point, and the three stage cascade structure is implemented to achieve tunable filtering.

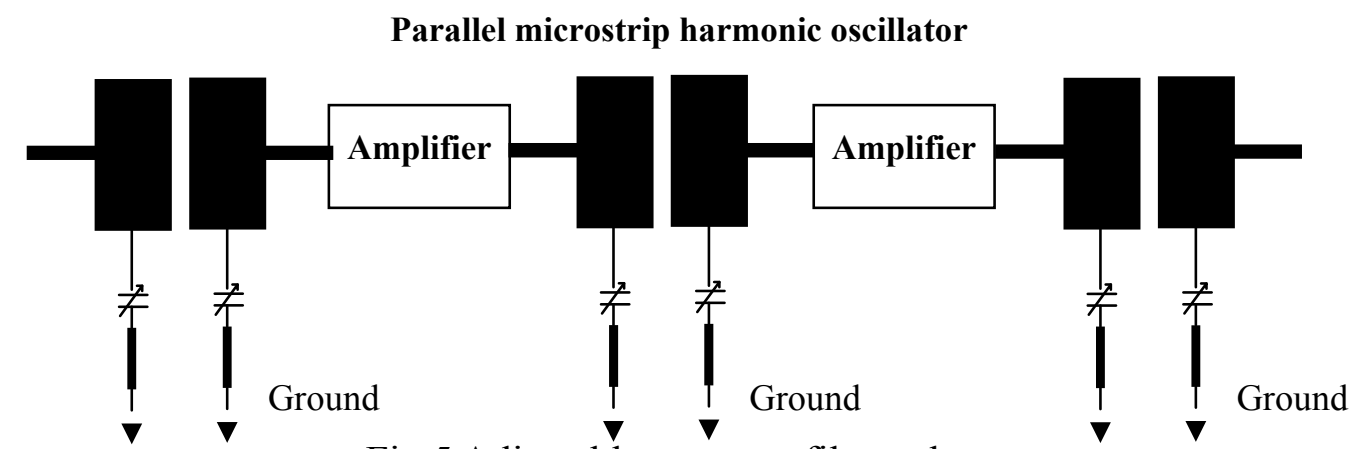

Fig.5 Adjustable resonant filter scheme

Full digital scanning control technology based on FPGA is the third key technology. Figure 6 is the automatic scan control logic, is the FPGA (field programmable large-scale integrated circuit) design and implementation, mainly including the scan timing control, RF switch automatic control, the first vibration frequency control, interface management, clock management and timing control circuit. The scan sequence control circuit generates the required synchronization signal, including high sweep signal (Hscan), main process signal (Buc), and child process signal (sBuc). The sBuc corresponds to each frequency and dwell time in each time sequence of VCO, and Buc corresponds to the pixel point of the display screen, while the Hscan signal is consistent with the simulation scan mode. The automatic control of the radio frequency channel switch automatically controls the automatic switching control of the RF channel and the high or low band channel switch. The first sweep frequency of vibration control is used to complete the refresh work of the variable frequency divider in the local oscillator loop circuit during the scan process.

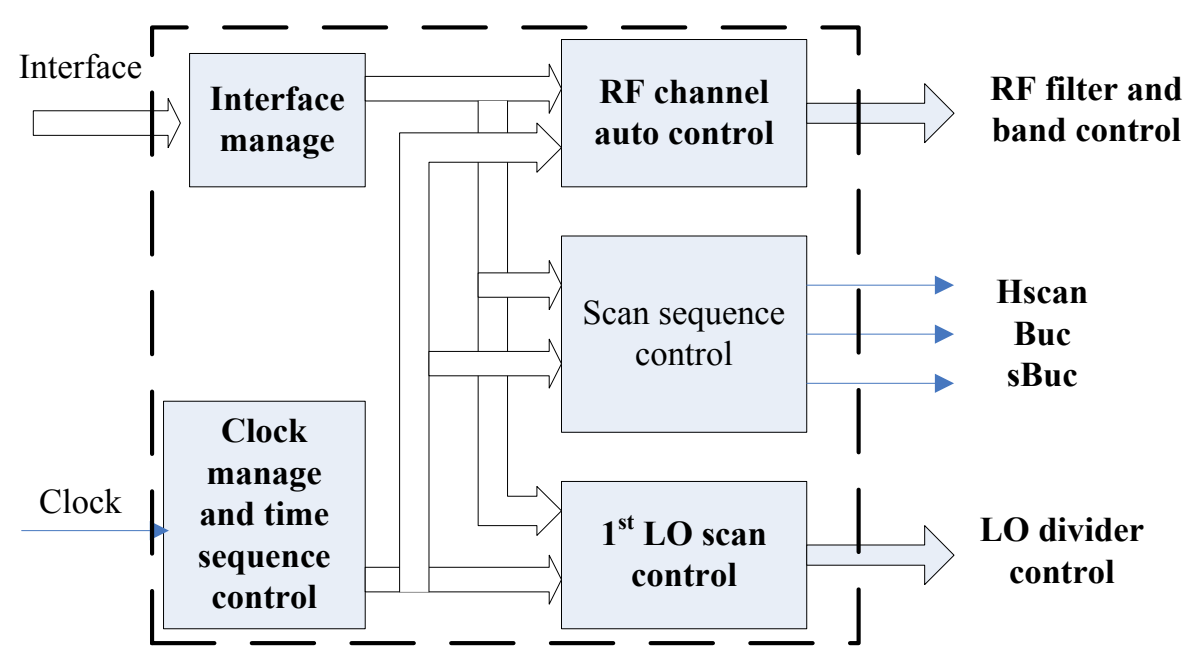

Fig.6 Scan control logic principle block diagram 


\section{Summary}

Based on the portable receiver, aiming at the advanced level of the world, fully learn from foreign advanced technology, the micro RF sensor is designed and realized.

The micro structure of micro broadband RF sensor is shown in Figure 7. The micro module structure design is adopted with small size, light weight, easy external field installation features, and it can meet the requirements of practical application, so it can be widely used in microwave communication, radio and television, radar, navigation, electronic warfare, space technology, satellite ground station, signal detection and other aspects of radio frequency signal monitoring and measurement, also it can really play a good premise and foundation in the grid monitoring and management system.

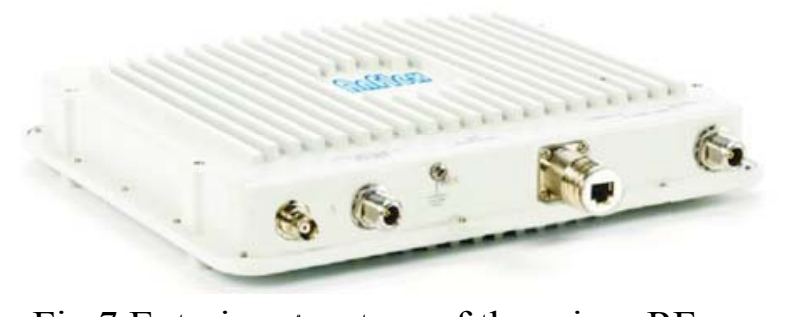

Fig. 7 Exterior structure of the micro RF sensor

\section{References}

[1] $\mathrm{Xu}$ shi-min, $\mathrm{Yu}$ hong-bing and $\mathrm{Chu}$ fei-huang, Battlefield situation cognition system basing on big electronic reconnaissance data processing, CMEEE 2014. 1-4.

[2] Tai ning, Zhang xiao-fa, Miniature digital frequency measure receiver, Electronic Design Engineering, 2013(5):117-119

[3] Sun xiao-hong, Research of grid radio monitoring system, Information Technology, 2013(8):136-140

[4] Mou jian-ming, The intermediate frequency digital receiver baseband design and implementation, Xidian University, 2012(4)

[5] Wang fu-rong, The analysis and application of gridding radio monitoring and management, Zhejiang University of Technology, 2012(3) 